\title{
AIDS-related Pneumocystis carinii Pneumonia with Disappearance of Cystic Lesions after Treatment
}

\author{
Mitsuru Konishi, Manabu Amimoto, Eiichiro Yoshimoto, Ken TaKahashi, Kei Mori, \\ Keiichi Mikasa and Nobuhiro Narita*
}

\begin{abstract}
A 21-year-old hemophiliac with human immunodeficiency virus (HIV) infection was admitted to our hospital because of bilateral pneumothoraces associated with Pneumocystis carinii pneumonia (PCP). He underwent chest tube drainages and intravenous pentamidine therapy, resulting in clinical improvement. Two months after treatment for PCP, cystic lesions that had existed before treatment disappeared on chest computed tomography. We concluded that Pneumocystis carinii infection might be associated with lung destruction and cyst formation, and that inflammatory exudates in the small bronchioles might act as a ball-valve with subsequent spontaneous pneumothoraces.
\end{abstract}

(Internal Medicine 41: 896-898, 2002)

Key words: HIV infection, pneumothorax, pentamidine, inhalation

\section{Introduction}

Pneumocystis carinii pneumonia (PCP) is one of the most common respiratory infections in patients with acquired immunodeficiency syndrome (AIDS). Pneumothorax is recognized as a complication that occurs frequently in patients with AIDS-related PCP. The etiological mechanism of pneumothorax is rupture of pulmonary cystic lesions associated with PCP, however, the etiology of cyst formation remains incompletely understood. We present a case in whom bilateral pneumothoraces associated with $\mathrm{PCP}$ occurred and describe the interesting course after treatment.

\section{Case Report}

A 21-year-old man, who had been diagnosed as having hemophilia A in 1980, was infected with human immunodefi- ciency virus (HIV) through the transfusion of non-heat-treated blood products. He started to receive prophylactic aerosolized pentamidine (300 mg every four weeks via an ultrasonic nebulizer) from May 1995 because his blood CD4 cell count declined in spite of antiretroviral therapy (zidovudine plus didanosine). He was admitted with a three-day history of nonproductive cough and persistent fever on January 9, 1997. He was a non-smoker and had rarely consumed alcohol. He had received no treatment except zidovudine, didanosine and aerosolized pentamidine. On admission to our hospital, he was 172 $\mathrm{cm}$ tall and weighed $53 \mathrm{~kg}$. Systemic blood pressure was 94/64 $\mathrm{mmHg}$ and his temperature was $37.5^{\circ} \mathrm{C}$. He had thrush on his oral mucosa and showed decreased breath sounds bilaterally. Laboratory data on admission showed a leukocyte count of $2,200 / \mu 1$, a CD4 cell count of $11 / \mu \mathrm{l}$, serum lactate dehydrogenase of $561 \mathrm{IU} / l$, and C-reactive protein of $6.4 \mathrm{mg} / \mathrm{dl}$. Arterial blood gas analysis while breathing room air demonstrated $\mathrm{PaO}_{2}$ of 79.8 torr and $\mathrm{PaCO}_{2}$ of 43.1 torr.

At the time of admission, the chest roentogenogram showed simultaneous bilateral spontaneous pneumothoraces (Fig. 1). After bilateral chest tubes were inserted, the expanded lungs revealed muliple cysts with a very mild infiltrate on chest computed tomography (CT)(Fig. 2). Fiberoptic bronchoscopy with bronchoalveolar lavage (BAL) was performed after improvement of the pneumothoraces. Since the BAL fluid smear stained with Grocott demonstrated the presence of Pneumocystis carinii cysts (Fig. 3), intravenous pentamidine therapy ( $4 \mathrm{mg} / \mathrm{kg} /$ day) was started. This therapy was continued for three weeks and pneumothorax did not recur, hence he left our hospital on February 21,1997 . After the discharge, he continued to receive prophylactic treatment with intravenous pentamidine $(150 \mathrm{mg}$ every two weeks) and has not had recurrent PCP. Two months after the discharge, chest CT showed disappearance of the cystic lesions (Fig. 4).

\section{Discussion}

PCP is recognized as a severe complication in patients with AIDS. Recently, the clinical manifestations of HIV infection

From the Second Department of Internal Medicine, Nara Medical University, Kashihara and *Nara Kouseikai Hospital, Yamato-kouriyama

Received for publication September 7, 2001; Accepted for publication February 26, 2002

Reprint requests should be addressed to Dr. Mitsuru Konishi, the Second Department of Internal Medicine, Nara Medical University, 840 Shijou Kashihara, Nara 634-8522 


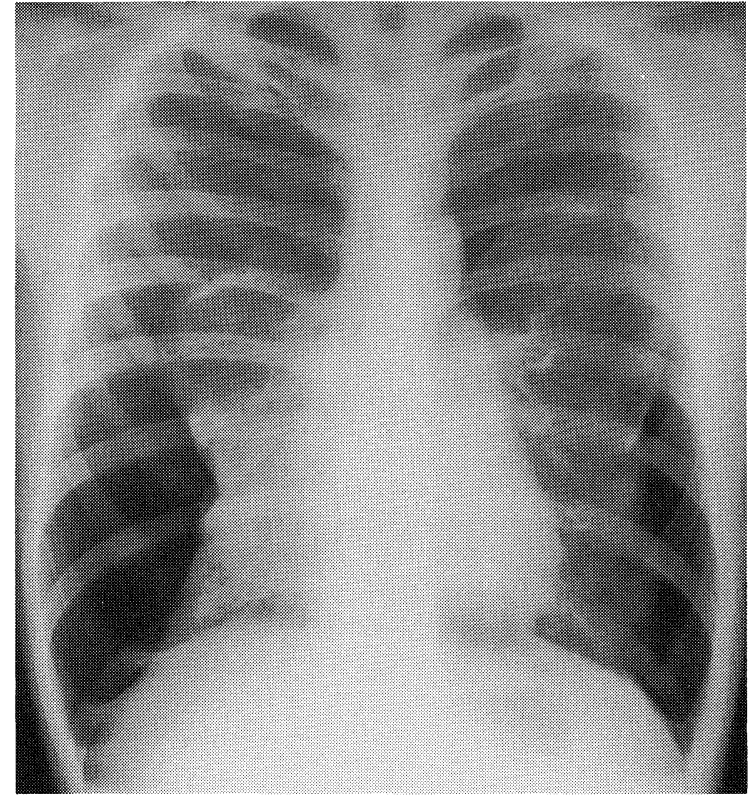

Figure 1. Chest roentgenogram showed bilateral pneumothoraces on admission.

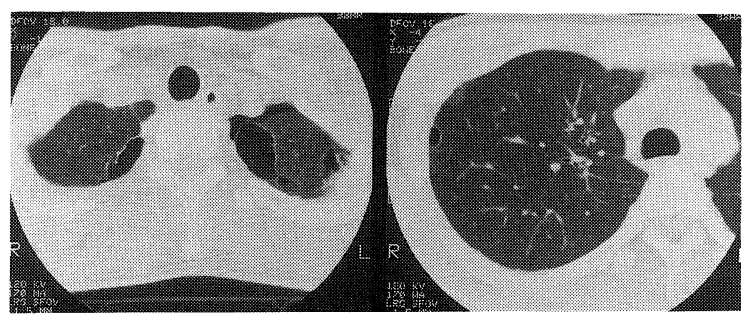

Figure 2. Chest computed tomography showed muliple cysts with a very mild infiltrate after chest tube drainages.

have changed dramatically with the increasing use of PCP prophylaxis and highly active antiretroviral therapy. Approximately $85 \%$ of all patients with advanced HIV disease have developed PCP once during the course of their disease when management of HIV infection was inadequate. The incidence of PCP among HIV-infected patients with fewer than 100 CD4 cells $/ \mu 1$ has declined markedly since 1996 (1). The hospital mortality rate for patients with HIV-related PCP has ranged from $60 \%$ in the late 1980 s to $10 \%$ recently (2). However, at present, PCP still remains an important opportunistic infection in patients with AIDS.

Chest roentgenograms are helpful in diagnosing PCP. The classic chest radiographic presentation of PCP is a bilateral perihilar or diffuse interstitial pattern, which may be finely granular, reticular or ground-glass in appearance. However, a

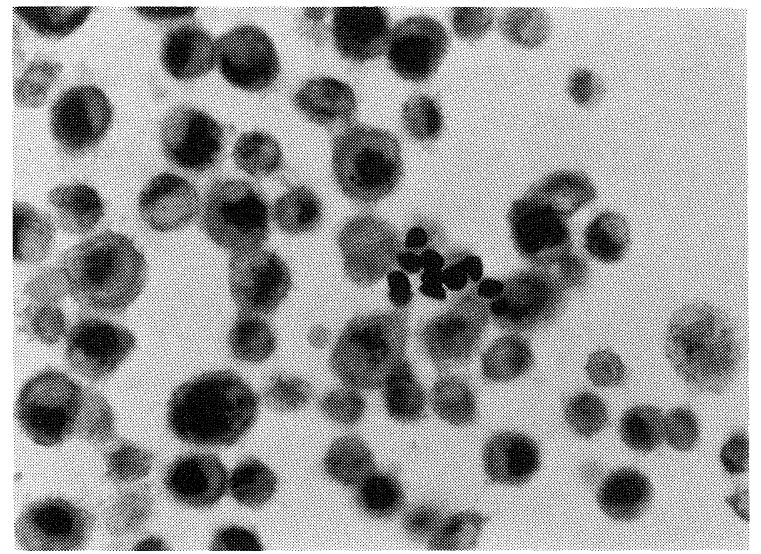

Figure 3. Grocott staining of a bronchoalveolar lavage fluid smear shows cysts of Pneumocystis carinii $(\times 1,000)$.

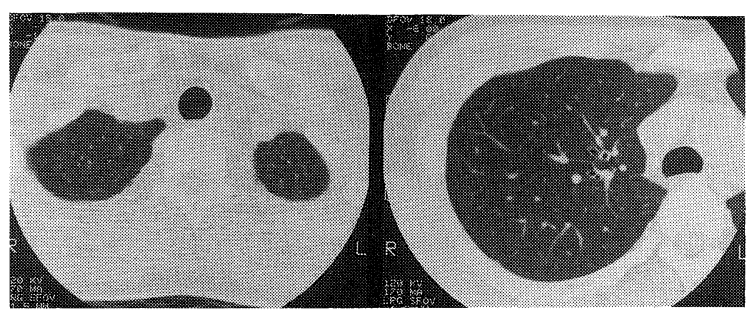

Figure 4. Chest computed tomography showed disappearance of cystic lesions approximately two months after treatment for Pneumocystis carinii pneumonia.

few cases have normal or subtle radiographic findings at the time of presentation. Chest CT, particularly high-resolution CT, is more sensitive than chest radiography for detecting PCP. The classic high-resolution CT finding is extensive ground-glass attenuation, which is often distributed in a patchy or geographic fashion (3). The chest CT manifestations of AIDS-related PCP are varied, so there are a few cases with a normal or near normal chest CT findings. In contrast, several radiographic features of PCP that were once considered unusual should now be considered as typical manifestations. These features include cystic lesions and spontaneous pneumothorax. The prevalence of cysts on chest roentgenograms or CT scan ranges from approximately $10 \%$ to $34 \%$ in HIV-infected patients with PCP. Cystic PCP is associated with an increased incidence of spontaneous pneumothorax (4). Chow and colleagues reported that 
$35 \%$ of patients with cystic PCP develop pneumothorax (5). The present case had mild hypoxemia because the chest radiographic finding was near normal except for cystic formation and pneumothoraces.

Spontaneous pneumothorax in a patient with AIDS was first documented in 1984 (6), and various reports have demonstrated the causative relation between PCP and spontaneous pneumothorax (7-10). Pastores et al reported that 67 patients (4.9\%) of 1,360 patients with AIDS and PCP were identified with spontaneous pneumothorax (7). Metersky et al indicated that a history of cigarette smoking, aerosol pentamidine treatment and the observation of cysts on chest radiography were associated with risk factors of spontaneous pneumothorax (8). The present case had two risk factors of pneumothorax: prophylactic pentamidine inhalation and observation of multiple cysts on chest CT.

The pathogenetic mechanisms involved in the development of PCP-related cysts and the association with pneumothorax have not yet been clarified. Various mechanisms have been proposed to date (7-11). Since the present case had prophylaxis with aerosol pentamidine, we predicted that pentamidine deposition in the lung periphery might be inadequate, thereby allowing the chronic and low-grade Pneumocystis carinii infection to persist, with subsequent lung destruction and cyst formation. Abd and coworkers measured the regional distribution in the lung of a mixter of technetium-99m diethylenetriaminentaacetic acid and pentamidine administered by means of aerosol to a patient and investigated the diminished deposition of aerosolized pentamidine in the upper lobes of lung (12). The present case disclosed PCP-related cysts in the bilateral upper lobes for that reason. Furthermore, because the cysts disappeared after treatment for PCP in this case, we presumed that inflammatory exudates in small bronchioles might act as a ball-valve and lead to cystic dilatation with subsequent spontaneous pneumothorax. Therefore, we consider that the treatment of PCP should be started early in advanced HIV-infected patients with cystic lesions of chest radiography due to the possibility that spontaneous pneumothorax may occur by ballvalve-induced cystic dilatation. Because the reason for the failure of prophylaxis against PCP may be related to an inadequate pulmonary concentration of pentamidine, a regimen of $300 \mathrm{mg}$ of aerosolized pentamidine administered twice monthly should be selected rather than $300 \mathrm{mg}$ once monthly (13).
Spontaneous pneumothorax associated with AIDS and PCP is often refractory or recurrent. The mortality rate is $18 \%$ to $24 \%$ in several reports $(7-10)$. Therefore, the treatment for pneumothorax includes chemical pleurodesis, and thoracoscopic or surgical treatment in addition to chest tube drainage. Metersky et al reported that a team approach (primary care physician, pulmonologist, and thoracic surgeon) employed early in the course of hospitalization of patients with AIDS-related pneumothorax would be advantageous (8).

\section{References}

1) Palella FJ Jr, Delaney KM, Moorman AC, et al. Declining morbidity and mortality among patients with advanced human immunodeficiency virus infection. N Engl J Med 338: 853-860, 1998.

2) Arozullah AM, Yarnold PR, Weinstein RA, et al. A new preadmission staging system for predicting inpatient mortality from HIV-associated Pneumocystis carinii pneumonia in the early highly active antiretrovial therapy (HAART) era. Am J Respir Crit Care Med 161: 1081-1086, 2000.

3) Kuhlman JE. Pneumocystic infection: The radiologist's perspective. Radiology 198: 623-635, 1996.

4) Boiselle PM, Crans CA Jr, Kaplan MA. The changing face of Pneumocystis carinii pneumonia in AIDS patients. Am J Roentgenol 172: 1301-1309, 1999.

5) Chow C, Templeton PA, White CS. Lung cysts associated with Pneumocystis carinii Pneumonia: radiographic characteristics, natural history, and complications. Am J Roentgenol 161: 527-531, 1993.

6) Wollschlager CM, Khan FA, Chitkara RK, Shivaram U. Pulmonary manifestations of the acquired immunodeficiency syndrome (AIDS). Chest 85: 197-202, 1984

7) Pastores SM, Garay SM, Naidich DP, Rom WN. Pneumothorax in patients with AIDS-related Pneumocystis carinii pneumonia. Am J Med Sci 312: 229-234, 1996.

8) Metersky ML, Colt HG, Olson LK, Shanks TG. AIDS-related spontaneous pneumothorax: Risk factors and treatment. Chest 108: 946-951, 1995.

9) Coker RJ, Moss F, Peters B, et al. Pneumothorax in patients with AIDS. Respir Med 87: 43-47, 1993.

10) Spivak H, Keller S. Spontaneous pneumothorax in the AIDS population. Am Surg 62: 753-756, 1996.

11) Feuerstein IM, Archer A, Pluda JM, et al. Thin-walled cavities, cysts, and pneumothorax in Pneumocystis carinii pneumonia: Further observations with histopathologic correlation. Radiology 174: 697-702, 1990.

12) Abd AG, Nierman DM, Ilowite JS, Pierson RN Jr, Bell ALL Jr. Bilateral upper lobe Pneumocystis carinii pneumonia in a patient receiving inhaled pentamidine prophylaxis. Chest 94: 329-331, 1988.

13) Golden JA, Katz MH, Chernoff DN, Duncan SM, Conte JE Jr. A randomized comparison of once-monthly or twice-monthly high-dose aerosolized pentamidine prophylaxis. Chest 104: 743-750, 1993. 